\title{
Luxação anterior recorrente do ombro após traumatismo com fratura apófise coracoide - Uma associação rara*
}

\section{Recurrent Anterior Shoulder Dislocation After Trauma with Coracoid Apophysis Fracture-A Rare Association}

\author{
Filomena Ferreira110 Ricardo Branco ${ }^{10}$ Rómulo Silva $^{10}$ Margarida Areias ${ }^{10}$ Carolina Oliveira ${ }^{10}$ \\ Norberto Silva1(10
}

${ }^{1}$ Departamento de Ortopedia e Traumatologia, Unidade Local de Saúde Alto Minho, Viana do Castelo, Portugal

Endereço para correspondência Filomena Ferreira, MD, Interno de Ortopedia e Traumatologia da Unidade Local de Saúde Alto Minho,

Rev Bras Ortop

Viana do Castelo, Portugal (e-mail: afilomenaf@gmail.com).

\begin{abstract}
Resumo
As luxações anteriores representam cerca de $96 \%$ do total de luxações do ombro, sendo a recidiva/instabilidade mais comum em pacientes jovens. A lesão de outras estruturas do ombro é frequente, nomeadamente a lesão óssea de Bankart. Contudo, a associação com a fratura da apófise coracoide é muito rara.

Este artigo descreve o caso clínico de um homem de 67 anos que recorreu ao serviço de urgência com queixas de omalgia persistente, com episódios de agudização, iniciados após queda da própria altura. O paciente apresentava ainda histórico de trauma do ombro 3 meses antes, avaliado em outro hospital. A luxação anterior do ombro foi constatada radiograficamente, e a tomografia computorizada (TC) do ombro confirmou erosão óssea da vertente anteroinferior da glenoide (perda óssea de cerca de $50 \%$ do diâmetro anteroposterior na região inferior da glenoide), com reabsorção quase completa de lesão óssea de Bankart (aparente em análise a posteriori da radiografia do episódio traumático inicial). Associadamente, foi diagnosticada uma fratura transversa da apófise coracoide (tipo II da classificação de Ogawa). O paciente foi submetido ao tratamento cirúrgico, com confecção do batente ósseo anterior utilizando remanescente do fragmento fraturado do coracoide suplementado por enxerto autólogo

Palavras-chave

- luxação do ombro

- fraturas do ombro

- processo coracoide tricortical do ilíaco, fixados com parafusos canulados (de acordo com as técnicas de Bristow-Latarjet e Eden-Hybinett).

No seguimento pós-operatório, foi observado um bom resultado funcional, sem novos episódios de luxação e sem queixas álgicas significativas.
\end{abstract}

Trabalho desenvolvido no Departamento de Ortopedia e Traumatologia da Unidade Local de Saude Alto Minho, Viana do Castelo, Portugal. recebido

27 de Maio de 2020

aceito

01 de Dezembro de 2020
DOI https://doi.org/

10.1055/s-0041-1724088.

ISSN 0102-3616. (c) 2021. Sociedade Brasileira de Ortopedia e Traumatologia. All rights reserved.

This is an open access article published by Thieme under the terms of the Creative Commons Attribution-NonDerivative-NonCommercial-License, permitting copying and reproduction so long as the original work is given appropriate credit. Contents may not be used for commercial purposes, or adapted, remixed, transformed or built upon. (https://creativecommons.org/ licenses/by-nc-nd/4.0/)

Thieme Revinter Publicações Ltda., Rua do Matoso 170, Rio de Janeiro, RJ, CEP 20270-135, Brazil 
Lesão óssea de Bankart e fratura da apófise coracoide Ferreira et al.

\begin{abstract}
Keywords

- shoulder dislocation

- shoulder fractures

- coracoid process

Anterior dislocations represent about $96 \%$ of total shoulder dislocations, with recurrence/instability being more common in young patients. Injury of other shoulder structures is frequent, namely bony Bankart lesion. However, the association with coracoid apophysis fracture is very rare.

The present article describes the clinical case of a 67-year-old man who presented to the emergency department with complaints of persistent omalgia, with acute episodes, beginning after a fall from his own height. The patient also presented history of shoulder trauma 3 months earlier, which was evaluated at another hospital. Shoulder anterior dislocation was observed radiographically, and the computed tomography (CT) confirmed bone erosion of the anteroinferior part of the glenoid (bone loss of about $50 \%$ of the anteroposterior diameter in the lower region of the glenoid), with almost complete resorption of the bony Bankart lesion (apparent in later analysis of the radiography of the initial traumatic episode). Connectedly, a transverse fracture of the coracoid apophysis (type II in the Ogawa classification) was diagnosed. The patient was submitted to surgical treatment, with anterior bone block confection using the remnant of the fractured fragment of the coracoid supplemented by tricortical autologous iliac graft, fixed with cannulated screws (according to the Bristow-Latarjet and Eden-Hybinett techniques).

In the postoperative follow-up, a good functional result was observed, with no new episodes of dislocation and no significant pain complaints.

A rare association of shoulder lesions is described, and the challenge of their treatment is highlighted, given the late diagnosis, as in the case presented.
\end{abstract}

Descreve-se uma associação rara de lesões do ombro, e salienta-se o desafio do tratamento das mesmas dado o seu diagnóstico tardio, como no caso apresentado.

\section{Introdução}

As luxações anteriores representam cerca de $96 \%$ do total de luxações do ombro. A recidiva de luxação é muito frequente em pacientes jovens, podendo ocorrer em até $80 \%$ dos casos. ${ }^{1}$ Contudo, em pacientes acima dos 40 anos, a recidiva ocorre em apenas cerca de $10 \%{ }^{2}$ Nos casos de luxação recidivante, é frequente ocorrer lesão das estruturas estabilizadoras da articulação glenoumeral (estáticas e/ou dinâmicas), sendo a lesão do labrum anteroinferior da glenoide com componente ósseo (lesão óssea de Bankart) uma sequela bem conhecida. ${ }^{3}$ O tratamento cirúrgico deste tipo de lesão varia entre a fixação da lesão de Bankart até à criação de aumentos ósseos anteriores utilizando apófise coracoide, enxerto autólogo ou heterólogo. ${ }^{4}$

As fraturas da apófise coracoide são pouco frequentes e de difícil diagnóstico, constituindo cerca de 3 a 13\% das fraturas da omoplata, que, por sua vez, representam menos de $1 \%$ do total de fraturas. ${ }^{5}$

A maioria das fraturas da coracoide descritas ocorrem na sequência de convulsões, sendo a sua associação com episódios de luxação anterior do ombro extremamente rara, existindo muito poucos relatos sobre o tratamento efetuado.

Este trabalho descreve o caso de um paciente com luxação recorrente/instabilidade do ombro consequente a lesão óssea de Bankart e fratura da apófise coracoide diagnosticadas tardiamente e as suas implicações no tratamento realizado.

\section{Caso clínico}

O caso trata de um paciente de 67 anos, do sexo masculino, sem antecedentes patológicos relevantes, nomeadamente epilepsia.

O paciente foi avaliado pelos autores no serviço de urgência (SU), por agudização de omalgia direita persistente, já com múltiplos episódios prévios semelhantes de agravamento da dor. A omalgia teve início 2 meses antes da apresentação aos autores, após queda da própria altura com traumatismo do membro superior em abdução e extensão, tendo sido avaliada em outra instituição. Não houve ocorrência de episódios traumáticos posteriormente.

O paciente apresentava-se com o membro superior suspenso em posição antálgica em ligeira rotação externa com sinal cabide positivo.

A luxação anterior do ombro foi verificada radiologicamente na data de avaliação pelos autores (-Figura 1), e a redução de luxação anterior foi então realizada.

Objetivamente com grande instabilidade do ombro, sendo fácil a redução de luxação e testes de apreensão anterior e "load and shift test" positivos. Sem déficits neurológicos.

A tomografia computorizada (TC) do ombro confirmou importante erosão óssea da vertente antero-nferior da glenoide, com perda óssea de cerca de $50 \%$ do diâmetro 


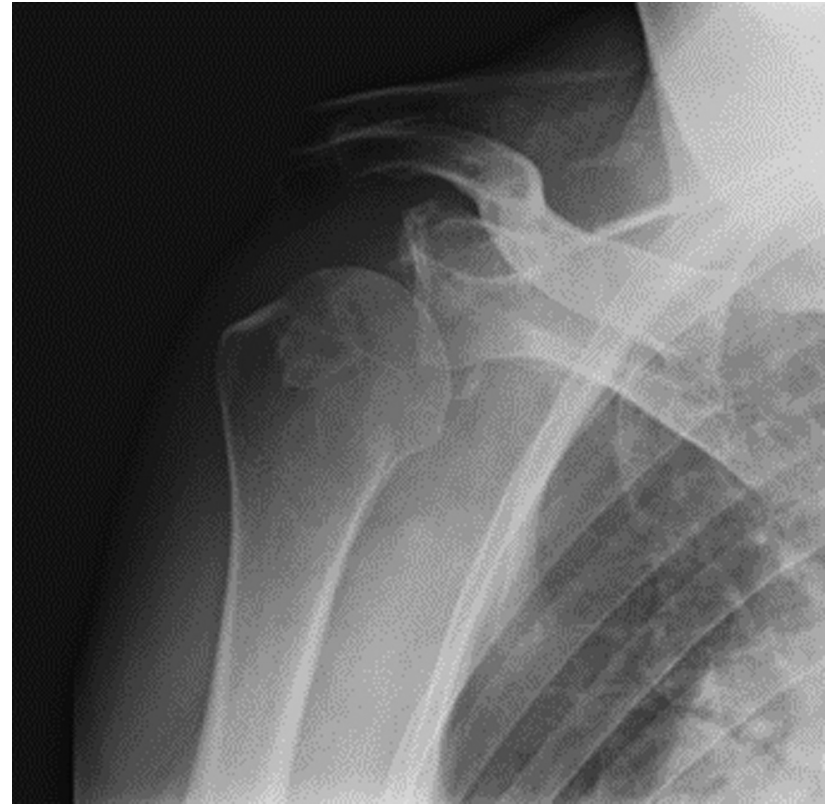

Fig. 1 Luxação anterior do ombro (radiografia do episódio de serviço de urgência usado na avaliação pelos autores).

anteroposterior na região inferior da glenoide (-Figura 2) e depressão focal infracentimétrica na vertente posteroexterna da cabeça umeral (lesão Hill-Sachs).

Em análise a posteriori da radiografia convencional do episódio traumático inicial (previamente avaliada em outra instituição), foi verificado fragmento de lesão óssea de Bankart, contudo sem luxação anterior do ombro (por provável redução espontânea). Na TC realizada pelos autores, foi observada reabsorção óssea quase completa do fragmento ósseo de Bankart. (-Figura 2).

Associadamente, foi diagnosticada fratura transversa da apófise coracoide (do tipo II na classificação de Ogawa), com reabsorção parcial do fragmento.

Dada a instabilidade com episódios recorrentes de luxação e dor intensa, foi proposto tratamento cirúrgico. Foi realizada uma abordagem deltopeitoral para exploração da articulação glenoumeral, com exérese de corpos livres intraarticulares e foi confirmado fragmento, de reduzidas dimensões, do coracoide no tendão conjunto.

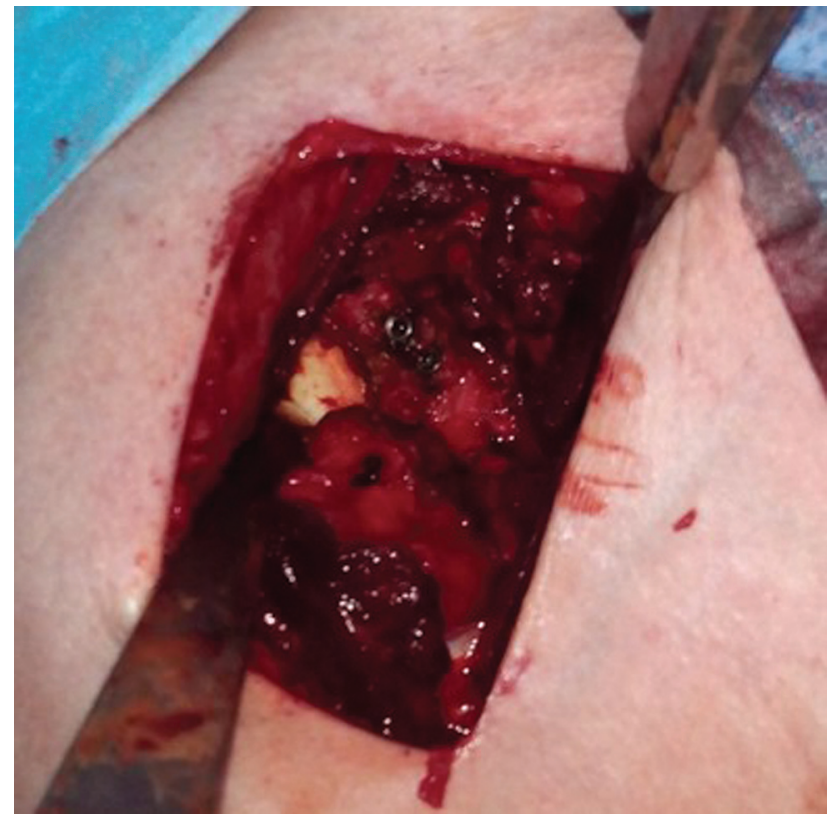

Fig. 3 Imagem intraoperatória de fixação de fragmento da coracoide e enxerto tricortical ilíaco à vertente anteroinferior da glenoide.

Foi confeccionado um batente ósseo anterior utilizando remanescentes do fragmento fraturado do coracoide (fixado com parafuso canulado no rebordo anteroinferior da glenoide - às 5h), suplementado por osteossíntese de enxerto tricortical colhido do ilíaco ipsilateral ( $\operatorname{com} 2,5 \mathrm{~cm} \times 3 \mathrm{~cm}$ ) com 2 parafusos nos 2/3 inferiores da glenoide (-Figuras 3 e 4 ).

O pós-operatório procedeu sem complicações. 0 paciente cumpriu 4 semanas de imobilização e posterior tratamento fisiátrico.

No seguimento pós-operatório, foi verificado um bom resultado funcional, sem novos episódios de luxação e sem queixas álgicas significativas. O escore na escala Disabilities of the Arm, Shoulder and Hand (DASH) antes da cirurgia foi de 51,6 e 1 ano após cirurgia foi de 18,3.

Um ano após cirurgia, o arco de movimento com limitação de 2 vértebras de rotação interna, era de $20^{\circ}$ de rotação externa e elevação anterior ativa de $100^{\circ}$. 0 teste de apreensão anterior e posterior, o "load and shift test," e o teste do sulco foram negativos.
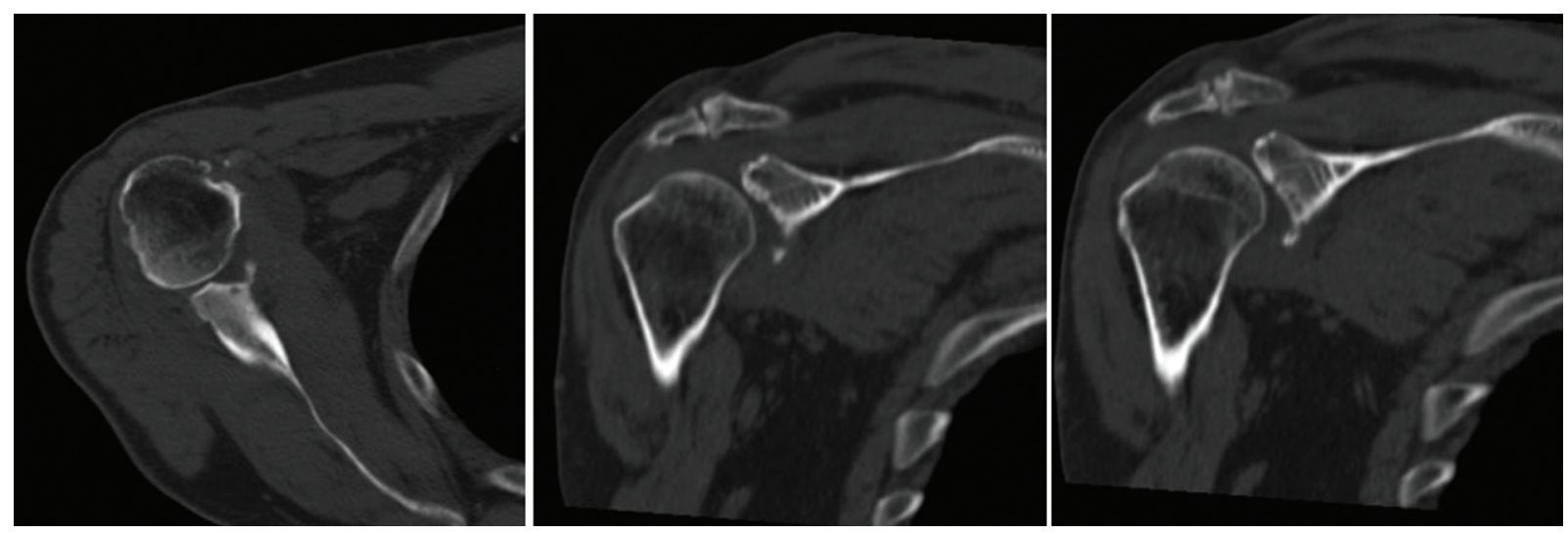

Fig. 2 Imagens de tomografia computadorizada - Lesão óssea de Bankart de cerca de 50\% da porção anteroinferior da glenoide com fragmentação óssea. 


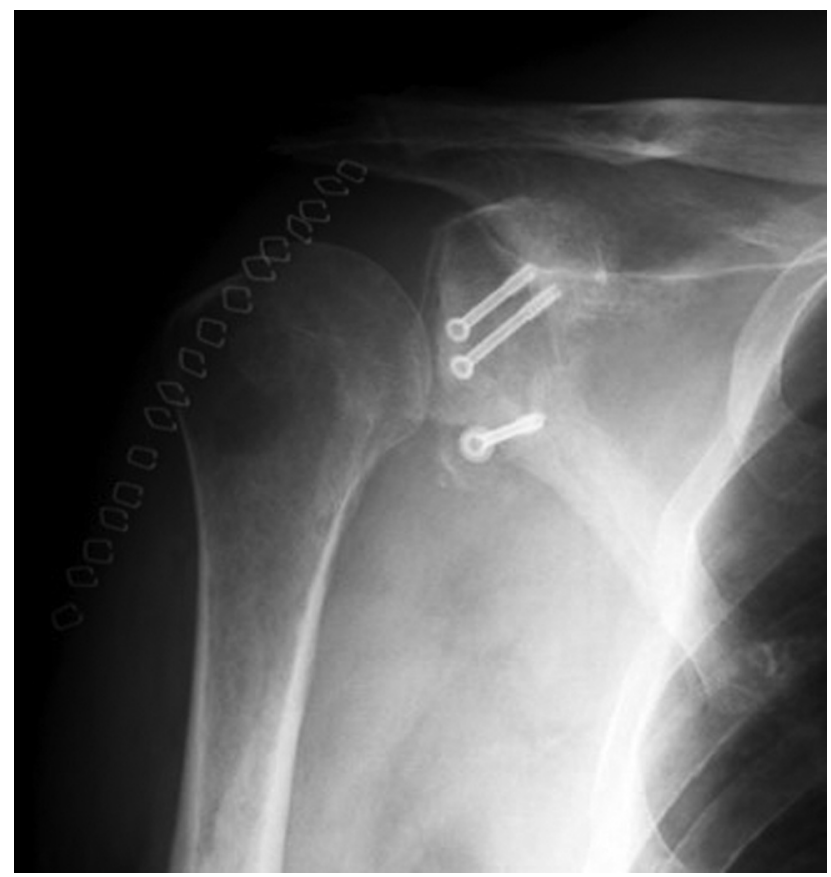

Fig. 4 Radiografia do ombro após a cirurgia.

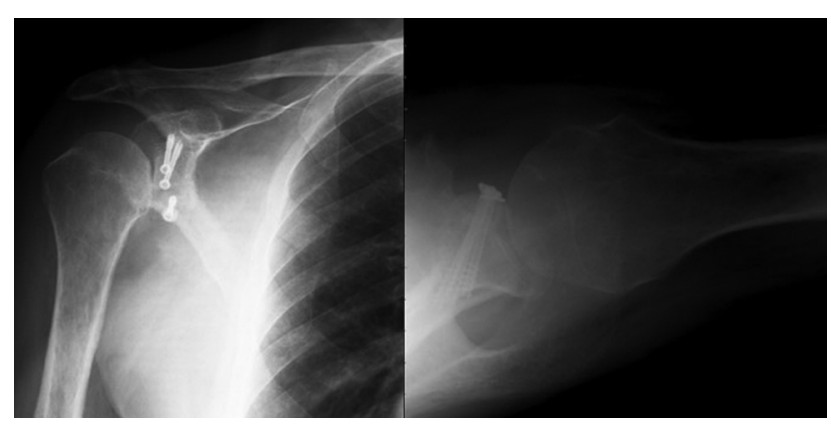

Fig. 5 Radiografia do ombro 1 ano após a cirurgia.

Radiograficamente, foram observados fragmentos ósseos consolidados na glenoide, mantendo adequado posicionamento dos parafusos canulados. Foram ainda observadas alterações degenerativas ligeiras da articulação glenoumeral, sobretudo na porção inferior (grau I da classificação Samilson e Prieto) (-Figura 5).

\section{Discussão}

No caso descrito, são vários os fatores que dificultam a criação de estabilidade no ombro:

1. A extensa destruição óssea da glenoide (cerca de $50 \%$ do diâmetro anteroposterior na porção inferior da glenoide) pela lesão óssea de Bankart e a progressão da erosão pelos repetidos episódios de luxação encontram-se bem documentadas como fatores de aumento de instabilidade e dificuldade no tratamento. ${ }^{6}$

2. O fragmento ósseo de Bankart encontrava-se inviável para osteossíntese devido à sua reabsorção e fragmentação desde o episódio traumático inicial até o diagnóstico.
3. A fratura do coracoide, com reabsorção de parte substancial do fragmento ósseo, tornava a criação de batente ósseo com a coracoide insuficiente para isoladamente criar estabilidade no ombro deste paciente.

Neste contexto, os autores optaram por combinar as técnicas de Bristow-Latarjet e de Eden-Hybinett já descritas. Assim, obteve-se estabilidade glenoumeral pela associação do efeito de batente ósseo e aumento da superfície articular da glenoide criados pelo fragmento de enxerto tricortical ilíaco, ${ }^{7}$ com o efeito estabilizador mecânico da apófise coracoide e também importante efeito estabilizador dinâmico do tendão conjunto. ${ }^{8}$

As lesões osteocartilagíneas resultantes das luxações recorrentes prévias e a congruência não perfeitamente anatômica da glenoide após a cirurgia levam a alterações degenerativas progressivas, sobretudo na cabeça umeral. Esta complicação é mais comum nos procedimentos de Eden-Hybinett, comparativamente à técnica de BristowLatarjet isoladamente. ${ }^{9}$ Neste caso, o grau de osteoartrose um ano após cirurgia é coincidente com o descrito na literatura para os graus I e II da classificação Samilson e Prieto, contudo com períodos de seguimento variáveis, dificultando a comparação. ${ }^{6}$

A estabilidade alcançada no paciente descrito é corroborada pela baixa taxa de recorrência de luxação após tratamento cirúrgico com aumento ósseo anterior. A incidência descrita é de até cerca de 4.9\% (4 dos 102 pacientes, todos associados a episódios traumáticos ou convulsões, após cirurgia de Latarjet). ${ }^{6,10}$ Não se encontrou qualquer descrição na literatura da taxa de recidiva com a associação de procedimentos descrita.

Este caso relata uma associação pouco comum de fraturas, salientando a importância de um elevado índice de suspeição clínica inicial e de observação radiológica minuciosa para o diagnóstico de todas as lesões presentes.

Além disso, reporta-se uma associação de técnicas cirúrgicas cujo sucesso terapêutico, apesar de se encontrar bem documentado isoladamente, merece maior estudo quando usado de forma conjugada para corroborar os bons resultados obtidos neste caso, podendo ser um procedimento a levar em conta em casos futuros semelhantes.

\section{Conflito de interesses}

Os autores declaram não haver conflito de interesses.

\section{Referências}

1 Colbenson K, Henry PDG, Kuhn JE. The True Recurrence Rate and Factors Predicting Recurrent Instability After Nonsurgical Management of Traumatic Primary Anterior Shoulder Dislocation: A Systematic Review. Arthrosc J Arthrosc Relat Surg 2018;32(12): 2616-2625

2 Owens BD, Duffey ML, Nelson BJ, DeBerardino TM, Taylor DC, Mountcastle SB. The incidence and characteristics of shoulder instability at the United States Military Academy. Am J Sports Med 2007;35(07):1168-1173

3 Patel RM, Amin NH, Lynch TSMA, Miniaci A. Management of bone loss in glenohumeral instability. Orthop Clin North Am 2014;45 (04):523-539 
4 Lavery KP, Mchale KJ, Rossy WH, Sanchez G, Provencher MT. Bony Augmentation for Anterior and Posterior Glenohumeral Instability in the Contact Athlete. Oper Tech Sports Med 2018;24(04):300-309

5 McGinnis M, Denton JR. Fractures of the scapula: a retrospective study of 40 fractured scapulae. J Trauma 1989;29(11):1488-1493

6 Beran MC, Donaldson CT, Bishop JY. Treatment of chronic glenoid defects in the setting of recurrent anterior shoulder instability: a systematic review. J Shoulder Elbow Surg 2010;19(05):769-780

7 Montgomery WHJr, Wahl M, Hettrich C, Itoi E, Lippitt SB, Matsen FA III. Anteroinferior bone-grafting can restore stability in osseous glenoid defects. J Bone Joint Surg Am 2005;87(09):1972-1977
8 Yamamoto N, Muraki T, An KN, et al. The stabilizing mechanism of the Latarjet procedure: a cadaveric study. J Bone Joint Surg Am 2013;95(15):1390-1397

9 Longo UG, Loppini M, Rizzello G, Ciuffreda M, Maffulli N, Denaro V. Latarjet, Bristow, and Eden-Hybinette procedures for anterior shoulder dislocation: systematic review and quantitative synthesis of the literature. Arthroscopy 2014;30(09):1184-1211

10 Burkhart SS, De Beer JF, Barth JR, Cresswell T, Roberts C, Richards DP. Results of modified Latarjet reconstruction in patients with anteroinferior instability and significant bone loss. Arthroscopy 2007;23(10):1033-1041 